\title{
A Joint NICER and XMM-Newton View of the "Magnificent" Thermally Emitting X-Ray Isolated Neutron Star RX J1605.3+3249
}

Malacaria, Christian; Bogdanov, Slavko; Ho, Wynn C. G.; Enoto, Teruaki; Ray, Paul S.; Arzoumanian, Zaven; Cazeau, Thoniel; Gendreau, Keith C.; Guillot, Sebastien; Guver, Tolga

Total number of authors:

12

Published in:

Astrophysical Journal

Link to article, DOI:

$10.3847 / 1538-4357 / a b 2875$

Publication date:

2019

Document Version

Publisher's PDF, also known as Version of record

Link back to DTU Orbit

Citation (APA):

Malacaria, C., Bogdanov, S., Ho, W. C. G., Enoto, T., Ray, P. S., Arzoumanian, Z., Cazeau, T., Gendreau, K. C., Guillot, S., Guver, T., Jaisawal, G. K., \& Wolff, M. T. (2019). A Joint NICER and XMM-Newton View of the "Magnificent" Thermally Emitting X-Ray Isolated Neutron Star RX J1605.3+3249. Astrophysical Journal, 880(2), [74]. https://doi.org/10.3847/1538-4357/ab2875

\section{General rights}

Copyright and moral rights for the publications made accessible in the public portal are retained by the authors and/or other copyright owners and it is a condition of accessing publications that users recognise and abide by the legal requirements associated with these rights.

- Users may download and print one copy of any publication from the public portal for the purpose of private study or research.

- You may not further distribute the material or use it for any profit-making activity or commercial gain

- You may freely distribute the URL identifying the publication in the public portal 


\title{
A Joint NICER and XMM-Newton View of the "Magnificent" Thermally Emitting X-Ray Isolated Neutron Star RX J1605.3+3249
}

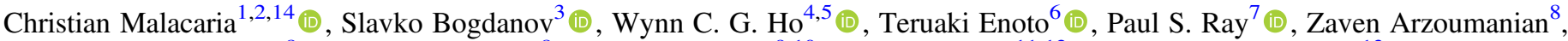 \\ Thoniel Cazeau $^{8}$, Keith C. Gendreau ${ }^{8}$, Sebastien Guillot ${ }^{9,10}$ (i), Tolga Güver ${ }^{11,12}$ (i), Gaurava K. Jaisawal ${ }^{13}$ (i), and \\ Michael T. Wolff ${ }^{7}$ (D) \\ on behalf of the NICER Magnetar \& Magnetospheres Team \\ ${ }^{1}$ NASA Marshall Space Flight Center, NSSTC, 320 Sparkman Drive, Huntsville, AL 35805, USA \\ ${ }^{2}$ Universities Space Research Association, NSSTC, 320 Sparkman Drive, Huntsville, AL 35805, USA \\ ${ }^{3}$ Columbia Astrophysics Laboratory, Columbia University, 550 West 120th Street, New York, NY 10027, USA \\ ${ }^{4}$ Department of Physics and Astronomy, Haverford College, 370 Lancaster Avenue, Haverford, PA 19041, USA \\ ${ }^{5}$ Mathematical Sciences, Physics and Astronomy, and STAG Research Centre, University of Southampton, Southampton SO17 1BJ, UK \\ ${ }^{6}$ Department of Astronomy, Kyoto University, Kitashirakawa-Oiwake-cho, Sakyo-ku, Kyoto 606-8502, Japan \\ ${ }^{7}$ Space Science Division, U.S. Naval Research Laboratory, Washington, DC 20375-5352, USA \\ ${ }^{8}$ X-ray Astrophysics Laboratory, Astrophysics Science Division, NASA's Goddard Space Flight Center, Greenbelt, MD 20771, USA \\ ${ }^{9}$ CNRS, IRAP, 9 avenue du Colonel Roche, BP 44346, F-31028 Toulouse Cedex 4, France \\ ${ }^{10}$ Université de Toulouse, CNES, UPS-OMP, F-31028 Toulouse, France \\ ${ }^{11}$ Department of Astronomy and Space Sciences, Science Faculty, Istanbul University, Beyazit, 34119 Istanbul, Turkey \\ ${ }_{13}^{12}$ Istanbul University Observatory Research and Application Center, Beyazit, 34119 Istanbul, Turkey \\ ${ }^{13}$ National Space Institute, Technical University of Denmark, Elektrovej, DK-2800 Lyngby, Denmark \\ Received 2019 January 20; revised 2019 May 28; accepted 2019 June 6; published 2019 July 26
}

\begin{abstract}
Thermally emitting X-ray isolated neutron stars (NSs) represent excellent targets for testing cooling surface emission and atmosphere models, which are used to infer the physical parameters of the NS. Among the seven known members of this class, RX J1605.3+3249 is the only one that still lacks confirmation of its spin period. Here we analyze NICER and XMM-Newton observations of RX J1605.3+3249, in order to address its timing and spectral behavior. Contrary to a previous tentative detection, but in agreement with the recent work by Pires et al., we find no significant pulsation with a pulsed fraction higher than $1.3 \%(3 \sigma)$ for periods above $150 \mathrm{~ms}$. We also find a limit of $2.6 \%$ for periods above $2 \mathrm{~ms}$, despite searches in different energy bands. The X-ray spectrum can be fit by either a double-blackbody model or by a single-temperature magnetized atmosphere model, both modified by a Gaussian absorption line at $\sim 0.44 \mathrm{keV}$. The origin of the absorption feature as a proton cyclotron line or as an atomic transition in the NS atmosphere is discussed. The predictions of the best-fit X-ray models extended to IR, optical, and UV bands, are compared with archival data. Our results are interpreted in the framework of a fallback disk scenario.
\end{abstract}

Key words: stars: individual (RX J1605.3+3249) - stars: neutron - X-rays: stars

\section{Introduction}

Isolated neutron stars (INSs) are a class of neutron stars (NSs) with no evidence of any stellar companion and an undetected or extremely weak radio counterpart (Kaspi et al. 2006). Among these is the group of thermally emitting INSs discovered by the ROSAT satellite, seven in number, and hence dubbed the "Magnificent Seven" (M7, Haberl 2007; Kaplan 2008; Turolla 2009), otherwise called thermally emitting X-ray isolated neutron stars (XINSs, Potekhin et al. 2015).

XINSs are thought to be members of the nearby $\mathrm{OB}$ associations of the Gould Belt (Walter 2001; Motch et al. 2003, 2005, 2009; Popov et al. 2003), located within a few hundreds of parsecs from the Sun (Posselt et al. 2007; van Kerkwijk \& Kaplan 2007). Their relatively low X-ray luminosity $\left(\sim 10^{31-32} \mathrm{erg} \mathrm{s}^{-1}\right)$ is consistent with cooling NSs of age $\sim 0.5 \mathrm{Myr}$ (Page et al. 2004), in rough agreement with estimates from their kinematic ages (Page et al. 2006; Haberl 2007; Motch et al. 2009). Their X-ray spectra are soft, thermal in nature, and usually fitted by blackbody components up to a few $\mathrm{keV}$ (e.g., Haberl 2007; van Kerkwijk \& Kaplan 2007). Atmospheric

\footnotetext{
${ }^{14}$ NASA Postdoctoral Fellow.
}

emission models have been proposed to fit the thermal emission from INSs (see Potekhin et al. 2016 and references therein); however, low-magnetic-field atmosphere models (Gänsicke et al. 2002; Zavlin \& Pavlov 2002) fail to reproduce the spectra of M7 members. On the other hand, for the highly magnetized atmosphere models, high-metallicity (e.g., iron) models predict a large number of absorption lines that are not observed, while pure hydrogen models reproduce spectral continua that are similar overall to the observed ones, and imply an effective temperature considerably lower than that suggested by blackbody models (Pavlov et al. 1996; Ho et al. 2007). Finally, while blackbody models underachieve the observed flux at optical wavelengths (Pavlov et al. 1996; Burwitz et al. 2003), atmospheric models overestimate it in some cases.

Timing studies of the M7 members in X-rays reveal spin periods in the range $3-17 \mathrm{~s}$ and relatively high magnetic fields, $B \sim 10^{13} \mathrm{G}$ (Haberl 2007; Kaplan et al. 2011; Hambaryan et al. 2017). Further similarity is observed among most M7 members, whose X-ray spectra show broad absorption features at energies $\sim 0.2-0.8 \mathrm{keV}$ (Haberl et al. 2003; Zane et al. 2005; Haberl 2007; Schwope et al. 2007; van Kerkwijk \& Kaplan 2007). Such features are usually attributed either to electron/proton cyclotron 
resonant scattering features (eCRSFs), proton cyclotron absorption features (pCFs), or to bound-bound/bound-free transitions in atoms of strongly magnetized NS hydrogen atmospheres (Zavlin \& Pavlov 2002; van Kerkwijk \& Kaplan 2007).

The only member of the M7 group that still lacks a coherent timing solution is RX J1605.3+3249 (J1605 hereafter; Schwope et al. 1999). A possible candidate spin period of 6.9 s was tentatively proposed by Haberl (2007), but was not confirmed in later observations. Another possible candidate spin period of $3.4 \mathrm{~s}$ (and a spin period derivative of $\dot{P} \sim 1.6 \times 10^{-12} \mathrm{~s} \mathrm{~s}^{-1}$ ) was proposed for $\mathrm{J} 1605$ by Pires et al. (2014), but it was significant only at a low confidence level $(\sim 4 \sigma)$.

The source distance has been analyzed in several works. Posselt et al. (2007) found two solutions for the distance, namely 390 or 325 pc. On the other hand, Motch et al. (1999) considered closer distance values, as low as $\sim 100 \mathrm{pc}$, while Motch et al. (2005) linked the source with the Sco OB2 association within the Gould Belt, at a mean distance of 120-140 pc. Tetzlaff et al. (2012) argued that J1605 was probably born in the Octans association from a supernova at $\approx 100 \mathrm{pc}$ and calculated the current distance of the NS as 300-400 pc.

A spectral feature at $\sim 0.45 \mathrm{keV}$ was first discovered by van Kerkwijk et al. (2004). Haberl (2007) found two additional absorption lines in the spectrum of J1605 obtained with XMMNewton, at energies of 0.59 and $0.78 \mathrm{keV}$, and consistent with energies in a 2:3:4 ratio. A narrow absorption feature at $0.58 \mathrm{keV}$ was also found by van Kerkwijk et al. (2004) and Hohle et al. (2012) using the high energy resolution instrument (RGS) on board XMM-Newton, with a width of $3.3 \mathrm{eV}$. Further analysis of XMM-Newton data by Pires et al. (2014) also found significant absorption lines in J1605 at slightly different energies than previous works, that is $0.44,0.58$, and $0.83 \mathrm{keV}$, while Pires et al. (2019) found no evidence of other absorption features than that at $\sim 0.4 \mathrm{eV}$.

In this work we report the results of recent observations of J1605 performed with XMM-Newton (XMM ) plus unpublished observations performed with the Neutron Star Interior Composition Explorer (NICER). We perform timing and spectral analysis in order to address open questions about J1605's main properties. Combining both NICER and XMM, our observations do not show evidence of pulsations in the $\mathrm{X}$-ray light curve of $\mathrm{J} 1605$. Moreover, our long NICER exposures allow us to perform the most sensitive pulse search down to $2 \mathrm{~ms}$ to date. The measured spectra are well fitted by a double-blackbody model or by a magnetized hydrogen atmospheric emission model, and we also confirm the presence of a broad absorption feature at $\sim 0.45 \mathrm{keV}$. Our timing and spectral results are consistent with the most recent work by Pires et al. (2019). We integrate archival IR/ Optical/UV data in our study, and interpret our findings in the context of the most recent XINS emission scenarios and the incidence of emission and absorption of their surrounding medium.

\section{Observations and Data Reduction}

In this work, we analyzed available NICER data for J1605, and only the most recent XMM data. The NICER and XMM observation log is reported in Table 1.
Table 1

Observations Log of RX J1605.3+3249

\begin{tabular}{|c|c|c|c|}
\hline Telescope & Obs ID & $\begin{array}{l}\text { Start Time } \\
\text { (UTC) }\end{array}$ & $\begin{array}{c}\text { Exposure }^{\mathrm{a}} \\
(\mathrm{s})\end{array}$ \\
\hline NICER & 1032020101 & 2017 Jul 19T22:46:40 & 115 \\
\hline NICER & 1032020102 & 2017 Jul 20T04:57:20 & 2620 \\
\hline NICER & 1032020103 & 2017 Jul 21T01:01:14 & 4545 \\
\hline NICER & 1032020104 & 2017 Jul 22T00:12:38 & 6038 \\
\hline NICER & 1032020105 & 2017 Jul 23T00:53:19 & 5924 \\
\hline NICER & 1032020106 & 2017 Jul 24T00:04:00 & 3184 \\
\hline NICER & 1032020107 & 2017 Oct 25T20:00:40 & 1111 \\
\hline NICER & 1032020108 & 2017 Oct 26T00:43:15 & 6324 \\
\hline NICER & 1032020109 & 2017 Oct $26 \mathrm{~T} 23: 52: 55$ & 7141 \\
\hline NICER & 1032020110 & 2017 Oct $28 \mathrm{~T} 00: 30: 41$ & 9187 \\
\hline NICER & 1032020111 & 2017 Oct $29 \mathrm{~T} 01: 12: 41$ & 8565 \\
\hline NICER & 1032020112 & 2017 Oct 30T00:21:42 & 10246 \\
\hline NICER & 1032020113 & 2017 Dec 01T02:03:10 & 2529 \\
\hline NICER & 1032020114 & 2017 Dec 02T02:50:35 & 2367 \\
\hline NICER & 1032020115 & 2017 Dec 03T00:27:35 & 10105 \\
\hline NICER & 1032020116 & 2017 Dec 04T01:09:16 & 3535 \\
\hline NICER & 1032020117 & 2017 Dec 05T00:10:11 & 1587 \\
\hline NICER & 1032020118 & 2017 Dec 06T02:29:17 & 6060 \\
\hline NICER & 1032020119 & 2017 Dec 07T01:43:20 & 9660 \\
\hline NICER & 1032020120 & 2017 Dec 08T00:50:43 & 16545 \\
\hline NICER & 1032020121 & 2017 Dec 08T23:58:00 & 14728 \\
\hline NICER & 1032020122 & 2017 Dec 10T00:41:16 & 10856 \\
\hline NICER & 1032020123 & 2017 Dec 10T23:55:30 & 4672 \\
\hline NICER & 1032020124 & 2017 Dec 19T00:44:31 & 2681 \\
\hline NICER & 1032020125 & 2017 Dec 19T23:47:40 & 7175 \\
\hline NICER & 1032020126 & 2017 Dec 21T02:02:20 & 2700 \\
\hline NICER & 1032020127 & 2017 Dec 23T00:32:19 & 2944 \\
\hline NICER & 1032020128 & 2017 Dec 24T01:01:53 & 822 \\
\hline NICER & 1032020129 & 2017 Dec 26T19:56:44 & 143 \\
\hline NICER & 1032020130 & 2017 Dec 27T23:17:00 & 574 \\
\hline NICER & 1032020131 & 2018 Apr 01T05:57:20 & 186 \\
\hline NICER & 1032020132 & 2018 Apr 08T03:25:20 & 308 \\
\hline$X M M$ & 0764460201 & $2015 \mathrm{Jul}$ 21T20:19:26 & 121353 \\
\hline$X M M$ & 0764460301 & 2015 Aug 20T18:07:20 & 68000 \\
\hline$X M M$ & 0764460401 & 2015 Aug 20T18:07:20 & 73000 \\
\hline$X M M$ & 0764460501 & 2016 Feb 10T22:36:56 & 62900 \\
\hline
\end{tabular}

Note.

${ }^{\mathrm{a}}$ Unfiltered times.

\subsection{NICER}

The NICER X-ray Timing Instrument (XTI, Gendreau et al. 2016) is an array of 56 co-aligned X-ray concentrator optics, each associated with a silicon drift detector sensitive in the $0.2-12 \mathrm{keV}$ band (Prigozhin et al. 2012). A single concentrator consists of 24 nested grazing-incidence goldcoated aluminum foil mirrors, parabolically shaped with a common focal length. To date, 52 detectors are operating, providing a peak effective area of $1900 \mathrm{~cm}^{2}$ at $\sim 1.5 \mathrm{keV}$, with an energy resolution of $\sim 100 \mathrm{eV}$ and a photon timetagging resolution of $\sim 100 \mathrm{~ns}$. With an effective area at $1 \mathrm{keV}$ of about $2000 \mathrm{~cm}^{2}$ (that is about two times that of $X M M$-pn and several times that of Chandra/ACIS), NICER perfectly matches the needs to analyze soft, thermal emission from NSs.

NICER observed J1605 for a total of 32 segments between 2017 July 19 (Obs ID: 1032020101) and 2018 April 8 (Obs ID: 103202032), collecting a total of $\sim 165 \mathrm{ks}$ of unfiltered 
exposure. The data were reduced using the software HEASOFT version 6.23 and NICERDAS version 2018-03-01_V004. ${ }^{15}$ Because of the low intrinsic source flux and the relatively high spectroscopic sensitivity needed for the purposes of the present work, accurate background removal is important. Good time intervals (GTIs) were first created by applying standard filtering (e.g., removing events detected during South Atlantic Anomaly passages). Then, further filtering was applied to remove high particle-radiation intervals associated with the Earth's auroral zones, i.e., "the polar horns," by applying a cut on the cutoff rigidity with COR_SAX $>4.0$. In addition, detectors flagged as "hot" by the data analysis software were removed for each observation. Then, GTIs were separated into times when NICER was exposed to direct sunlight (orbit day), and times when the satellite was within the Earth shadow (orbit night). This procedure deals with different background components separately, such as the optical loading prominent only during day orbits at energies below $\sim 0.35 \mathrm{keV}$. In this way lowenergy data are free from artificial structures, although at the expense of a considerable amount of exposure time. This does not represent the standard procedure for NICER data analysis but ensures a low background level in the energy range $\sim 0.3-0.5 \mathrm{keV}$, where an absorption feature is expected (see Section 1). Finally, a flat count-rate cut (at $\sim 10 \mathrm{c} \mathrm{s}^{-1}$ ) to the resulting $0.25-12 \mathrm{keV}$ light curve was applied to remove possible remaining background flaring events. The resulting final exposures are $58 \mathrm{ks}$ and $16 \mathrm{ks}$ for day and night orbits, respectively. The source spectra were grouped using the GRPPHA tool to have a minimum of 25 counts per bin. The most recent response files provided by the NICER instrumental calibration team were used. ${ }^{16}$

Background spectra were created from data acquired from one of seven "blank sky" targets based on the Rossi X-Ray Timing Explorer (RXTE) background fields (Jahoda et al. 2006). Among the seven available fields, we selected the target with the smallest angular separation from J1605 (BKGD \#8, $\Delta \theta \sim 45^{\circ}$ ). All observations of the background field were reduced as described above. The resulting final exposures are $10 \mathrm{ks}$ and $15 \mathrm{ks}$ for day and night background spectra, respectively.

Source counts are $1.5 \times 10^{5}$ and $2.2 \times 10^{5}$, corresponding to mean count rates of 5.9 and $3.6 \mathrm{~s}^{-1}$ for night and day spectra, respectively, and accounting for about $94 \%$ of the total.

\subsection{XMM-Newton}

XMM observed J1605 a total of four times between 2015 July 21 (Obs ID: 0764460201) and 2016 February 10 (Obs ID: 0764460501). In this work, we only use data from the $X M M$ European Photon Imaging Camera (EPIC) cameras-i.e., the PN CCD (Strüder et al. 2001) and the MOS CCDs (Turner et al. 2001) - both sensitive in the $0.15-12 \mathrm{keV}$ range and offering spectral resolution $E / \Delta E \sim 20-50$ at $6.5 \mathrm{keV}$ (see Pires et al. 2019, for analysis of RGS data). However, as J1605 has a soft spectrum, to avoid background contamination we restricted our analysis to the range $0.2-1.2 \mathrm{keV}$. The PN and MOS cameras were set in Full and Large Window modes with thin filters, respectively. The total unfiltered exposure was $\sim 325 \mathrm{ks}$. Data reduction was performed using the Science Analysis System (SAS) software xmmsas_20170719_1539-16.1.0, with the

\footnotetext{
15 https://heasarc.gsfc.nasa.gov/docs/nicer/nicer_analysis.html

16 Response Matrix File and Ancillary Response File version 1.02.
}

latest available calibration files. Step-by-step reduction was performed following the official SAS Science Threads. ${ }^{17}$ To remove high background flaring activity for EPIC cameras, single-event (PATTERN $==0$ ), high-energy light curves were extracted for each camera; then, a count-rate threshold was chosen corresponding to the low and steady background. Applying such a threshold to the light curves resulted in the selection of GTIs. For the pn-camera, single and double events were selected (patter $n \leqslant 4$ ), while single, double, triple, and quadruple events were accepted for the MOS cameras (pattern $\leqslant 12$ ). Background circular regions of size $60^{\prime \prime}$ to $100^{\prime \prime}$ were defined off-source, on the same chip as the target for the PN camera, while a different yet close and largely sourcefree chip for MOS was used to generate background spectra. PN and MOS spectra were extracted separately for each observation.

Because of the relatively high source flux, the thin filter, and the Full/Large Window observing mode, the observations are affected by pile-up at a few percent level in both the PN and MOS cameras. Even though the observed count rate is within "tolerant" levels according to Jethwa et al. (2015), we opted for a conservative approach in order to ensure a confident energy redistribution of the recorded events, thus helping constrain the spectral parameters and spectral features. To minimize the resulting spectral distortion, we excised the core of the pointspread function (PSF) in each observation and extracted counts in an annulus centered on the source, retaining only the lowercount-rate wings of the PSF. To optimize the excising radius, we excluded progressively larger radii of the PSF core (up to a radius of about $15^{\prime \prime}$ ), testing their impact on the pile-up reduction using the epatplot task ${ }^{18}$ until pile-up effects were negligible. This resulted in losses of about 10 and $20 \%$ of the original MOS and PN camera exposures, respectively.

We then used the SAS task epicspeccombine to combine spectra in order to improve statistics. However, we note that, according to the SAS team, the task can be used only to merge spectra and response files that have been generated in the same PI channel interval, and selecting a spectrum with a non-standard PI range results in the wrong response matrices and therefore unreliable spectra. ${ }^{19}$ For this reason, we merged all the different cameras' spectra separately, resulting in one merged PN spectrum and one merged MOS spectrum. The resulting final exposures are 285 and $280 \mathrm{ks}$ for MOS2 and MOS1 and $225 \mathrm{ks}$ for PN. Spectral bins have been grouped to have a minimum of 25 counts per spectral bin, while the maximum oversample of the instrumental energy resolution was fixed to a factor of 3 .

\section{Analysis}

\subsection{Pulsation Searches}

To search for a periodic pulsed signal associated with the rotation of the NS, the event detection times were first translated to the solar system barycenter with the barycorr tool in HEASOFT for NICER and the barycen task in SAS for $X M M$. For this purpose we adopted the DE405 solar system ephemeris and the position of J1605 derived from the XMM EPIC pn imaging data from the longest exposure (Obs ID 0764460201), R.A. $=16: 05: 18.48$, decl. $=+32: 49: 21.0$.

\footnotetext{
17 https://www.cosmos.esa.int/web/xmm-newton/sas-threads

18 https://www.cosmos.esa.int/web/xmm-newton/sas-thread-epatplot

19 https://www.cosmos.esa.int/web/xmm-newton/sas-thread-epic-merging
} 
The periodicity searches were conducted using the PRESTO Fourier-domain pulsar search software package (Ransom et al. 2002). The acceleration search technique implemented in PRESTO allowed us to coherently search long time series (up to several months) by considering a wide range of frequency drifts caused by a range of possible rotational spindown values of the NS. For XMM, only the EPIC PN data were used for this analysis, due to the significantly greater sensitivity and better time resolution $(\Delta t=73.4 \mathrm{~ms})$ of this instrument compared to the MOS cameras $(\Delta t=0.9 \mathrm{~s})$. In addition, just a subset of the NICER observations was used for the pulsation search-in particular, the observations taken 2017 July 19-24 (22.4 ks of unfiltered exposure), 2017 October 25-30 (42.4 ks unfiltered exposure), and 2017 December 1-27 (82.7 ks unfiltered exposure), as they provide the most compact set of deep exposures, which is desirable for sensitive coherent pulsation searches. These NICER subsets from July, October, and December data were first searched separately and also combined to perform a coherent search.

The XMM events were binned at the intrinsic EPIC pn $73.4 \mathrm{~ms}$ detector time resolution, while the events from NICER (which has an absolute time resolution of $\sim 100 \mathrm{~ns}$ ) were binned at a time resolution of $0.977 \mathrm{~ms}(1024 \mathrm{~Hz})$ for the separate searches of the 2017 July, October, and December subsets. To coherently search the combined October and December NICER event lists, we used a $0.0625 \mathrm{~s}$ binning, and to search the July-December data set, we used a time binning of $0.25 \mathrm{~s}$.

We first conducted periodicity searches over the $0.3-1.2 \mathrm{keV}$ band. However, as J1605 may exhibit a multi-temperature thermal spectrum, as suggested in previous works (e.g., Pires et al. 2014) and shown in Section 3.2, it is possible that pulsations may only arise from the hotter and smaller regions on the stellar surface. Alternatively, the pulsations of the cool and hot emission regions may be significantly out of phase such that pulsations are strongly suppressed when integrated over the full $0.3-1.2 \mathrm{keV}$ band (see, e.g., Gotthelf \& Halpern 2009, for the curious case of the Puppis A pulsar). To account for this scenario, we conducted additional searches for periodicity restricted to events in the $0.3-0.5 \mathrm{keV}$ and $0.8-1.2 \mathrm{keV}$ bands, where the cool and hot components dominate, respectively.

The XINSs RX J0720.4-3125 (Borghese et al. 2015) and RX J1308.6+2127 (Borghese et al. 2017) are known to exhibit narrow absorption features that only appear over a fraction of their rotation periods. In principle, the same could be the case for J1605 such that pronounced pulsations at the NS rotation period only occur in the absorption line. Motivated by this prospect, we conducted searches using only events in the energy range around the absorption feature apparent in the X-ray spectrum, $0.4-0.5 \mathrm{keV}$.

No statistically significant $\left(\geqslant 4 \sigma\right.$, as determined by the $Z_{n}^{2}$ test; see Buccheri et al. 1983) periodic signals are found in either the NICER or XMM data sets for any choice of energy band. From the $X M M$ data we can set a $3 \sigma$ upper limit of $1.3 \%$ on the pulsed fraction over the $0.3-1.2 \mathrm{keV}$ band, assuming a sinusoidal pulse, for spin periods greater than $0.1468 \mathrm{~s}$, comparable to what Pires et al. (2019) found. For the NICER data in the $0.3-1.2 \mathrm{keV}$ band, after accounting for the additional number of trials from the acceleration search, we obtain a pulsed fraction limit of $<2.6 \%$ for spin periods greater than $1.95 \mathrm{~ms}$ from the 2017 December observations, and a $<1.6 \%$ limit for periods greater than $0.125 \mathrm{~s}$ by combining the
2017 October and December observations. Including the NICER exposures from 2017 July 19-24 data does not result in a significant improvement in sensitivity to pulsations due to the three-month gap and shorter exposure relative to the October and December data, yielding a $<1.6 \%$ limit for periods $>0.5 \mathrm{~s}$. The $<2.6 \%$ limit (at $3 \sigma$ ) for short periods $(>2 \mathrm{~ms}$ ) is significantly stronger than the $3.2 \%-5.0 \%$ limits (at $2 \sigma$ ) for periods $>1.2$ ms obtained by van Kerkwijk et al. (2004) using $X M M$ pn fast-timing data (see in particular their Table 3).

The absence of pulsations in substantially deeper exposures indicates that the period reported in Pires et al. (2014) based on a $60 \mathrm{ks} X M M$ exposure was spurious, in agreement with Pires et al. (2019).

\subsection{Spectral Analysis}

Spectra were fitted using the XSPEC 12.10.0 package (Arnaud 1996). We fitted NICER (day and night) and XMM (pn and MOS) spectra simultaneously, allowing for a crossnormalization factor among the different spectra. The energy band for fitting was limited to $0.2-1.2 \mathrm{keV}$, above which the background dominates.

Photoelectric absorption model and elemental abundances were set according to Wilms et al. (2000) (tbabs in XSPEC) to account for photoelectric absorption by neutral interstellar matter (or column density $N_{\mathrm{H}}$ ), and assuming model-relative (wilm) solar abundances. During our spectral analysis we allowed the column density parameter to vary (although we also explored the case where the absorption column density was kept fixed to the Galactic value, $2.4 \times 10^{20} \mathrm{~cm}^{-2}$ ). This resulted in values larger than the Galactic $N_{\mathrm{H}}$ value to the source $\left(\sim(3-5) \times 10^{20} \mathrm{~cm}^{-2}\right)$, as well as larger than the value found in previous works (e.g., van Kerkwijk \& Kaplan 2007; Pires et al. 2014). This is similar to what Pires et al. (2019) also found, i.e., nominal values of the $N_{\mathrm{H}}$ parameter generally higher than Galactic (that is, in the range $(2.5-5.3) \times$ $10^{20} \mathrm{~cm}^{-2}$ ). Moreover, as discussed in Viganò et al. (2014), the single-blackbody model used in previous works to fit the X-ray data of J1605 (see Section 1) tends to underestimate $N_{\mathrm{H}}$ by $20 \%-30 \%$ with respect to the actual value. However, the higher than Galactic $N_{\mathrm{H}}$ value in the case of J1605 is related to the covariance with the parameters of the broad absorption line at $\sim 0.4 \mathrm{keV}$ and to our ignorance of the true continuum spectral shape. We also verified that employing different absorption models (e.g., wabs in XSPEC, see Morrison \& McCammon 1983) and abundances (e.g., angr in XSPEC, see Anders \& Grevesse 1989) returns $N_{\mathrm{H}}$ values consistent with those obtained using the tbabs model.

The continuum of J1605 is known to be fitted by an absorbed single- or double-blackbody model modified by at least one Gaussian absorption line at $0.45 \mathrm{keV}$ (van Kerkwijk et al. 2004; Pires et al. 2014) and we tested both models to fit our data. We used the bbodyrad model (instead of the simpler bbody) from XSPEC because it allows us to link the normalization $K$ of the blackbody component to the emitting surface area through the relation $K=R_{\mathrm{km}}^{2} / D_{10 \mathrm{kpc}}^{2}$, where $R_{\mathrm{km}}$ is the radius of the emitting surface in units of $\mathrm{km}$ and $D_{10 \mathrm{kpc}}$ is the distance from the source in units of $10 \mathrm{kpc}$. A singleblackbody model returns a reduced $\chi^{2}>5$ (for 488 dof). Our data are best fit by an absorbed double-blackbody model with an absorption feature at $\sim 0.43 \mathrm{keV}$ (see Table 2 and Figure 1). The absorption feature has been modeled with a Gaussian 
Table 2

Best-fit Results of J1605 Spectral Analysis with a Double-blackbody Model and Two Atmospheric Models, NSA and NSMAXG

\begin{tabular}{lccc}
\hline \hline & Double-BB & NSA $(g=2.43)$ & NSMAXG \\
\hline$N_{\mathrm{H}}\left[10^{20} \mathrm{~cm}^{-2}\right]$ & $4.6_{-1.4}^{+1.4}$ & $3.4_{-0.2}^{+0.2}$ & $5.6_{-0.3}^{+0.3}$ \\
$k T_{\text {cool }}[\mathrm{eV}]$ & $63_{-6}^{+7}$ & $\ldots$ & $\ldots$ \\
$k T_{\text {hot }}[\mathrm{eV}]$ & $119_{-4}^{+6}$ & $\ldots$ & $\ldots$ \\
$\log T_{\text {eff }}[\mathrm{K}]$ & $\ldots$ & $5.737_{-0.005}^{+0.005}$ & $5.729_{-0.022}^{+0.016}$ \\
$M_{\mathrm{NS}}\left[M_{\odot}\right]$ & $\ldots$ & 1.4 (fixed) & $2.04_{-0.49}^{+0.19}$ \\
$R_{\mathrm{NS}}[\mathrm{km}]$ & $\ldots$ & $10 \mathrm{~km}$ (fixed) & $15.6_{-0.79}^{+0.62}$ \\
$B\left[10^{13} \mathrm{G}\right]$ & $\ldots$ & 1 (fixed) & 1 (fixed) \\
$K_{\text {cold }}$ & $3.3_{-2.2}^{+8.0} \mathrm{E}+5$ & $\ldots$ & $\ldots$ \\
$K_{\text {hot }}$ & $1.9_{-0.8}^{+0.8} \mathrm{E}+3$ & $\ldots$ & $\ldots$ \\
Distance ${ }^{\mathrm{a}}[\mathrm{kpc}]$ & $0.174_{-0.079}^{+0.127}$ & $0.092_{-0.005}^{+0.005}$ & 0.1 (fixed) \\
$K_{\text {atmos }}$ & $\ldots$ & $1.19_{-0.12}^{+0.12} \mathrm{E}-4$ & 1.0 (fixed) \\
$E_{\text {abs }}[\mathrm{keV}]$ & $0.435_{-0.006}^{+0.013}$ & $0.452_{-0.003}^{+0.003}$ & $0.445_{-0.003}^{+0.003}$ \\
$\sigma_{\text {abs }}[\mathrm{keV}]$ & $0.110_{-0.011}^{+0.010}$ & $0.087_{-0.005}^{+0.004}$ & $0.092_{-0.004}^{+0.005}$ \\
$\tau_{\text {abs }} C_{\text {MOs }}{ }^{\mathrm{b}}$ & $0.24_{-0.08}^{+0.13}$ & $0.098_{-0.000}^{+0.009}$ & $0.133_{-0.016}^{+0.011}$ \\
$C_{\text {Night }}$ & $0.849_{-0.005}^{+0.006}$ & $0.849_{-0.005}^{+0.006}$ & $0.857_{-0.006}^{+0.006}$ \\
$C_{\text {Day }}{ }^{\mathrm{c}}$ & $0.848_{-0.013}^{+0.013}$ & $0.848_{-0.013}^{+0.013}$ & $0.858_{-0.013}^{+0.013}$ \\
Flux & $0.725_{-0.005}^{+0.005}$ & $0.725_{-0.005}^{+0.005}$ & $0.733_{-0.005}^{+0.005}$ \\
$\chi_{\text {red }}^{2} / \mathrm{dof}$ & $1.10_{-0.27}^{+0.42}$ & $1.08_{-0.03}^{+0.04}$ & $1.16_{-0.34}^{+0.40}$ \\
\hline
\end{tabular}

Notes. All reported errors are at the $90 \%$ confidence level. Parameters indicated with $K_{x}$ represent the normalization value of the cold and hot component for the double-blackbody model, and that of the atmospheric NSA and NSMAXG models.

${ }^{a}$ Distance $D$ of the source calculated as proportional, or equal to, $1 / K_{x}^{2}$ for the double-BB and NSA models, respectively (see the text), or reported as a parameter of the model for the NSMAXG model.

${ }^{\mathrm{b}}$ Cross-normalization factors for MOS, NICER night and day spectra, respectively. PN cross-normalization factor was kept fixed to unity.

${ }^{\mathrm{c}}$ Unabsorbed flux calculated for the continuum component(s) in the $0.3-1.2 \mathrm{keV}$ band and reported in units of $10^{-11} \mathrm{erg} \mathrm{cm}^{-2} \mathrm{~s}^{-1}$. Flux values with estimated errors were derived using the cflux model from XSPEC.

profile (the multiplicative gabs component in XSPEC):

$$
F(E)=\exp \left\{-\frac{\tau_{\mathrm{abs}}}{\sqrt{2 \pi} \sigma_{\mathrm{abs}}} \exp \left(-\frac{\left(E-E_{\mathrm{abs}}\right)^{2}}{2 \sigma_{\mathrm{abs}}^{2}}\right)\right\},
$$

where $E_{\text {abs }}$ is the line centroid energy, and $\tau_{\text {abs }}$ and $\sigma_{\text {abs }}$ are the optical depth and the width of the line, respectively. The second blackbody component and the absorption feature improve the reduced $\chi^{2}$ to a value of 2.4 and 1.1, respectively. The blackbody temperatures and absorption line energy are in general agreement with findings from Pires et al. (2019). On the contrary, previous works (e.g., Haberl 2007; Pires et al. 2014) require additional absorption features at $\sim 0.58$ and $0.8 \mathrm{keV}$. However, we note that the observation of spectral features in the spectra of XINS can be model-dependent, and can also depend on the exact details of the analysis. Moreover, previous results based on XMM observations of J1605 are now superseded by the Pires et al. (2019) analysis and data set. On the other hand, we notice that the results from Haberl (2007) were obtained using a single-temperature blackbody model and cannot therefore be compared to that used in the present work. Moreover, the fit obtained by Haberl (2007) is only marginally acceptable, with a reduced $\chi_{\text {red }}^{2} \sim 1.4$, and structured residuals resulting from the best-fit model.
The absorption feature found in previous works at $\sim 0.58 \mathrm{keV}$ (van Kerkwijk et al. 2004; Hohle et al. 2012; Pires et al. 2014) is narrow (Gaussian width in the range $\sim 3-16 \mathrm{eV}$ ) and needs the resolution power of instruments like RGS on board $X M M$ to be resolved, which is beyond the scope of the present work. However, we note that Pires et al. (2019) also found no evidence of the narrow absorption feature at $\sim 0.58 \mathrm{keV}$ in the most recent XMM-RGS observations.

Finally, we note that Schwope et al. (2007) find that the energy of the two absorption features observed in the XINS RBS 1223 spectrum considerably changes among different observations, with the lowest energy absorption feature changing from $0.39 \mathrm{keV}$ in 2003 to $0.20 \mathrm{keV}$ in 2005 and the higher-energy absorption feature going from undetected to $0.73 \mathrm{keV}$, respectively (while roughly harmonic in the rest of the observations). Therefore, the centroid energy of the detected features may vary on timescales of years (a somewhat analogous phenomenon is observed in accreting NSs, e.g., Her X-1 Staubert et al. 2016, although the accretion process in those sources is likely responsible for the long-term variation of the cyclotron line energy). More recent works (Hambaryan et al. 2011; Borghese et al. 2017) also find inconsistent line energies for RBS 1223, although the inconsistency might be due to the difference in the analysis approach.

More complex, physical models have also been tested, such as those representing the X-ray spectrum emitted from the atmosphere of a NS. Various NS atmospheric models have been proposed in the literature, and tested in the present work, e.g., the magnetic/non-magnetic versions of the fully ionized hydrogen atmosphere model (NSA in XSPEC, Pavlov et al. 1995; Zavlin et al. 1996), the non-magnetic hydrogen atmosphere model with variable surface gravitational acceleration (NSAGRAV in XSPEC, Zavlin et al. 1996), and the weakly/ strongly magnetized versions of the partially ionized atmospheric model that allows for variable surface gravitational acceleration and is composed of hydrogen $(\mathrm{H})$ or heavier elements (e.g., carbon, oxygen, iron, NSX and NSMAXG model in XSPEC, Ho \& Heinke 2009; Ho 2014). Among all tested atmospheric models, the only ones that returned an acceptable fit were (1) the NSA model in the magnetic case $\left(B=10^{13} \mathrm{G}\right)$; and (2) the NSMAXG model with $B=10^{13} \mathrm{G}$, the latter with the distance and normalization values kept fixed (see Table 2). None of the other tested combinations of the abovementioned atmospheric models returned a statistically acceptable fit or physically meaningful values of the model parameters, and will not be discussed further. Moreover, we stress that the source distance commonly adopted in the literature $(\sim 350 \mathrm{pc}$; see, e.g., Posselt et al. 2007) is nonetheless uncertain and cannot be readily accommodated by our data, while a possible distance of $\sim 150$ pc (see Section 1) is consistent with our data.

Similar to the double-blackbody model, the atmospheric model fits also require a Gaussian absorption line at $\sim 0.45 \mathrm{keV}$ (see Table 2 and Figure 2). Finally, because the NSA model is developed for a standard gravitational acceleration $g=2.43 \times$ $10^{14} \mathrm{~cm} \mathrm{~s}^{-2}$, corresponding to standard NS mass $\mathrm{M}_{\mathrm{NS}}=$ $1.4 M_{\odot}$ and radius $R_{\mathrm{NS}}=10 \mathrm{~km}$, these two parameters were kept fixed during the fitting procedure. ${ }^{20}$

Analogous spectral continuum models and spectral features have been employed by Pires et al. (2019) to fit the XMM data of J1605. Their spectral results are overall consistent with those

\footnotetext{
${ }^{20}$ https://heasarc.gsfc.nasa.gov/xanadu/xspec/manual/node196.html
} 


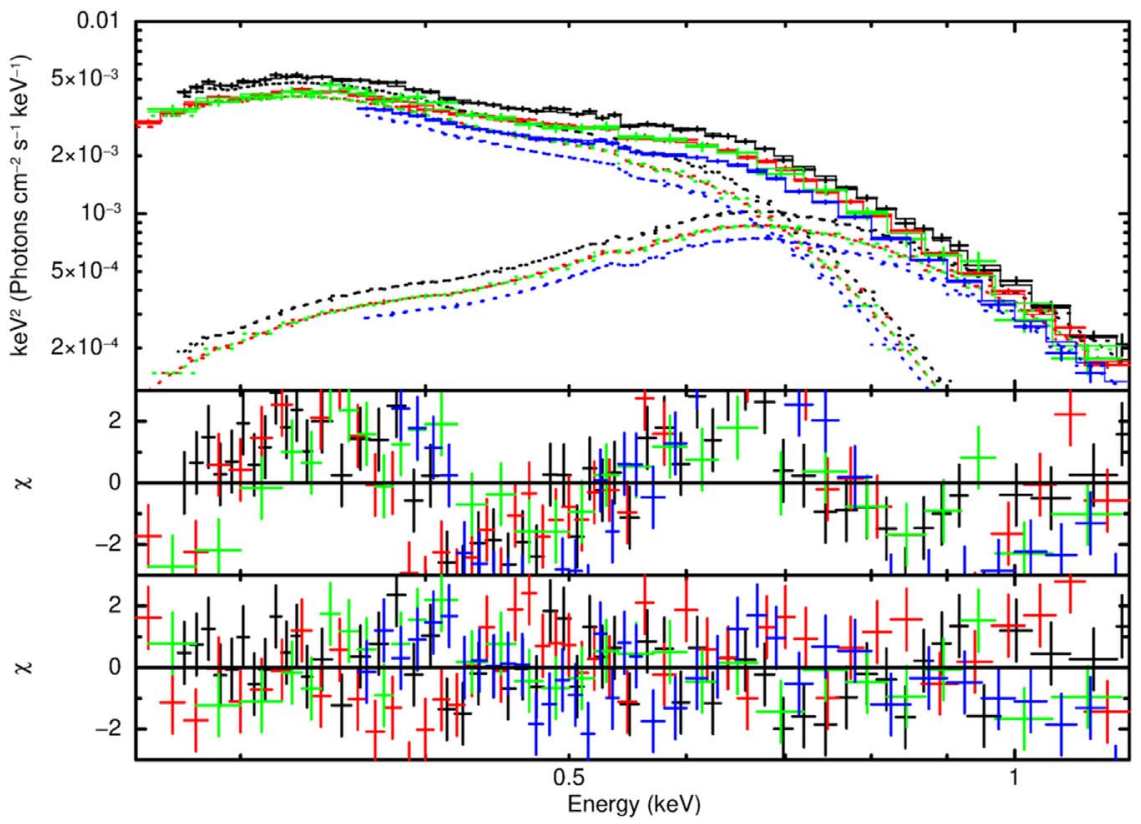

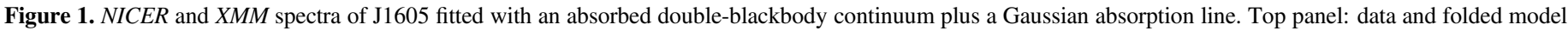

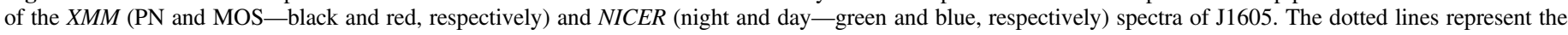

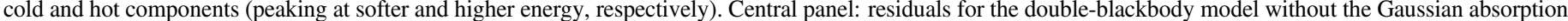
component. Bottom panel: residuals for the best-fit model. Spectra and residuals have been rebinned for plotting purposes.

found in the present work. However, their best-fit spectral models of EPIC data find an absorption feature at $385 \mathrm{eV}$, a significantly different energy than that found here, while the energy of the absorption feature found by the analysis of XMMRGS spectra is consistent with our results.

\section{Discussion}

\subsection{A Double-blackbody Model for the Continuum Emission}

The spectral continuum of XINS is broadly consistent with a single-temperature blackbody component $\left(k T_{b b} \sim 40-100 \mathrm{eV}\right)$ slightly modified by interstellar absorption $\left(N_{\mathrm{H}} \sim 10^{20} \mathrm{~cm}^{-2}\right)$. This result is generally interpreted as thermal emission from the NS surface. However, with the availability of high signal-tonoise spectra, deviations from that simple model emerge (see, e.g., Zane et al. 2011 and references therein). Phase-averaged as well as phase-resolved spectra from XINSs generally are better fitted by a combination of two or three blackbody components, physically interpreted as coming from different regions of the star surface. Such deviations from a purely single-temperature component are expected as the result of an inhomogeneous temperature distribution across the NS surface, expected from theoretical arguments and stemming from the presence of strong magnetic fields causing, e.g., anisotropic thermal conductivity and non-spherically symmetric magnetic field dissipation, leading to the presence of hot spots (Page et al. 2007; Pons et al. 2009). In particular, the origin of the double-blackbody continuum is generally attributed to two thermally emitting spots: a smaller hot spot, usually associated with the magnetic poles, and a larger cool spot as wide as the NS itself and due to the cooling surface.

Our analysis finds that a double-blackbody model fits well the continuum emission from J1605 (see Figure 1). The temperatures of the cold and hot regions are 63 and $119 \mathrm{eV}$, respectively, in general agreement with previous work on J1605 and other XINS. Assuming a $10 \mathrm{~km}$ radius NS for the cold component (see Section 4.3) we get a distance of $\sim 174_{-80}^{+127} \mathrm{pc}$, while the normalization of the hot component, given a nominal distance of $174 \mathrm{pc}$ returns a radius of the emitting hot spot equal to $\sim 0.76_{-0.18}^{+0.18} \mathrm{~km}$. The case of the double-blackbody model with the column density value fixed to the Galactic value $\left(N_{\mathrm{H}}=2.4 \times 10^{20} \mathrm{~cm}^{-2}\right)$ has also been investigated. This model fits well the X-ray data $\left(\chi_{\text {red }}^{2} /\right.$ dof $=$ $1.14 / 484)$ and returns a distance of $393 \pm 18 \mathrm{pc}$, more in line with previous works (Tetzlaff et al. 2012, and references therein, see also Section 1), while both the hot and cold blackbody components show about $10 \mathrm{eV}$ hotter temperature values.

Finally, to investigate deviations from a pure, singleblackbody continuum as found in Motch et al. (2005) for J1605, we compared that with the broadband spectral energy distribution obtained from our double-blackbody model. Our results are presented in Figure 3 . Below $\sim 0.5 \mathrm{keV}$, the flux predicted by the double-blackbody model is about 10 times higher than that predicted by the single-blackbody, while the hot blackbody component returns a spectrum that is roughly comparable to that of the single-blackbody model of Motch et al. (2005), considering the relatively small difference between the temperature values (119 and $99 \mathrm{eV}$, respectively). However, the cold component brings an important contribution to the soft $\mathrm{X}$-ray band $(\lesssim 0.5 \mathrm{keV})$, modifying the emerging spectrum at softer $\mathrm{X}$-ray energies.

An important consequence deriving from the inclusion of a cold component is the model prediction at lower wavelengths, namely in the IR/optical/UV band. Figure 3 compares the prediction of our model with Hubble Space Telescope (HST)/ Subaru optical/UV data from Kaplan et al. (2003), Motch et al. (2005), Kaplan et al. (2011), as well as WISE and Spitzer IR data (Posselt et al. 2014). Optical/UV magnitudes have been converted to flux according to the standard STDMAG conversion (Kaplan et al. 2003, 2011), and corrected to account for optical extinction following the empirical relation 


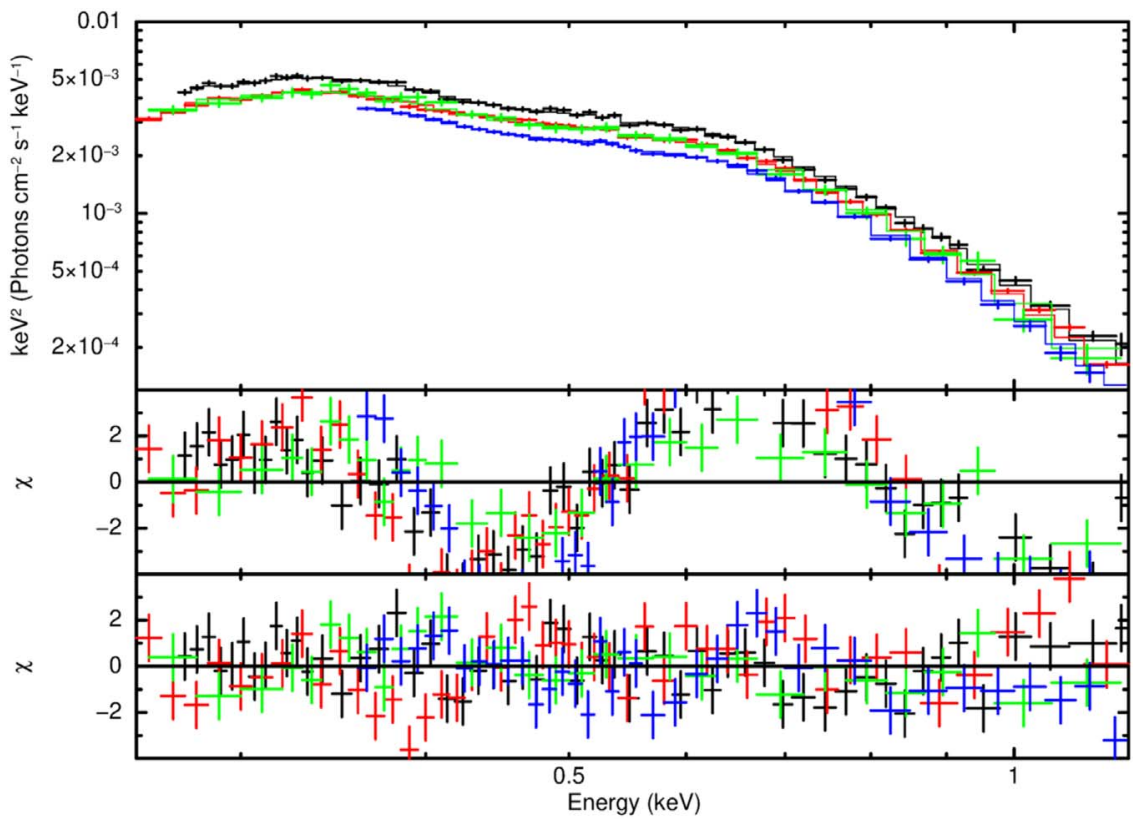

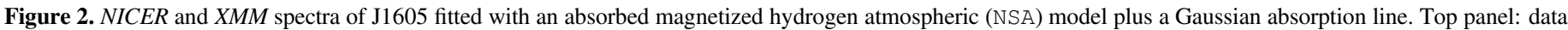

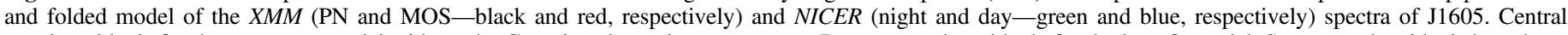

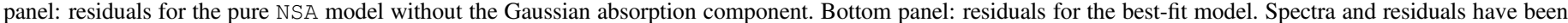
rebinned for plotting purposes.

described in Foight et al. $(2016), N_{\mathrm{H}}=(2.87 \pm 0.12) \times 10^{21}$ $\mathrm{A}_{\mathrm{V}} \mathrm{cm}^{-2}$ (which, however, suffers from considerable scatter at low $N_{\mathrm{H}}$ values such as those derived for J1605). The model can fit optical data from the $B$ to the $R$ bands without requiring additional components or conditions, such as a power-law component or a thin hydrogen atmosphere (Motch et al. 2003, 2005; Ho et al. 2007). This is a direct result of the inclusion of a second, colder blackbody component in the fit. However, as illustrated by Kaplan et al. (2011), J1605's optical/UV data show a trend that is less steep than a $\propto \lambda^{-4}$, blackbody-like function. As a result, the nominal doubleblackbody model fits the optical data but results in an overestimation of the UV flux, consistent with it only being within the large uncertainty (see Figure 3).

\subsection{The Fallback Disk Scenario}

As outlined in Section 4.1 and Figure 3, the best-fit doubleblackbody model is subject to further considerations. If the physical interpretation of the double-blackbody model is plausible, and only the nominal predicted values are considered, then a mechanism must be at work to suppress the inferred flux in the UV band. A possible mechanism responsible for the suppression of the UV flux in XINS is potential fallback disks or dusty belts surrounding the compact object, which are, at least in some cases, indicated as a distinct possibility (Perna et al. 2000; Posselt et al. 2014, 2018). When present, dust grains around the NS are heated by the UV radiation, for which the grains behave as nearly perfect absorbers, thus reradiating the absorbed flux in the infrared band. However, the double-blackbody model predicts a flux that is $\sim 3$ times larger than that observed in UV (see Figure 3), which requires absorption values of a few times $10^{21} \mathrm{~cm}^{-2}$, that is about 10 times the Galactic value to the source, and many times the amount observed in our best-fit model (see Table 2). Assuming that the fallback disk is distributed as a torus with inner and outer radii of $10^{10}$ and $10^{16} \mathrm{~cm}$, respectively (see
Posselt et al. 2014), a column density of $3 \times 10^{21} \mathrm{~cm}^{-2}$ corresponds to a torus mass of about $30 M_{\oplus}$. The IR emission from such a massive torus would therefore be intense, e.g., according to Equation (1) in Posselt et al. (2014), of the order of $10^{2} \mathrm{mJy}$ at $160 \mu \mathrm{m}$ and for a source distance of $173 \mathrm{pc}$. However, Posselt et al. (2014) found no significant infrared counterpart at the position of $\mathrm{J} 1605$, a result that puts upper limits on the IR emission from this source, $<12.2 \mathrm{mJy}$ at $160 \mu \mathrm{m}$ (that is, approximately $18 \mathrm{keV} \mathrm{s}^{-1} \mathrm{~cm}^{-2} \mathrm{keV}^{-1}$ at $8 \times 10^{-6} \mathrm{keV}$, see Figure 3 for comparison) and on the possible disk mass, $<2.2 M_{\oplus}$. Therefore, a dusty fallback disk surrounding the NS seems unsuitable to explain the UV suppression implied by the double-blackbody model. Alternatively, the best-fit double-blackbody model with the column density value fixed to the Galactic value can be considered. Figure 3 shows that this model is consistent with the UV data point (at $\sim 10^{-2} \mathrm{keV}$ ), but not with the optical data. This result can be interpreted in terms of the model employed by Ertan et al. (2017), where the optical flux is emitted mainly from the inner rim of the fallback disk in the form of a blackbody spectrum. In Figure 3 we show a blackbody model with $k T_{\text {eff }} \sim 1.3 \mathrm{keV}$ and optical flux $F_{\text {opt }} \sim 10^{-3} F_{\mathrm{X}}$, where $F_{\mathrm{X}}$ is the X-ray flux. These values are typical of XINS (Ertan et al. 2017) and result to fit well the optical data leaving the UV flux almost unaffected.

\subsection{An Atmospheric Model for the Continuum Emission}

Besides the double-blackbody model, X-ray spectra from $\mathrm{J} 1605$ analyzed in this work are equally well fit by specific configurations of the atmospheric NSA and NSMAXG models (see Table 2 and Figure 2). In our analysis, both models assume constant magnetic field strength and temperature across the NS surface. While one could expect magnetic field (and temperature) variation across the emission region, the non-detection of pulsations (see Section 3.1) supports our assumption. 


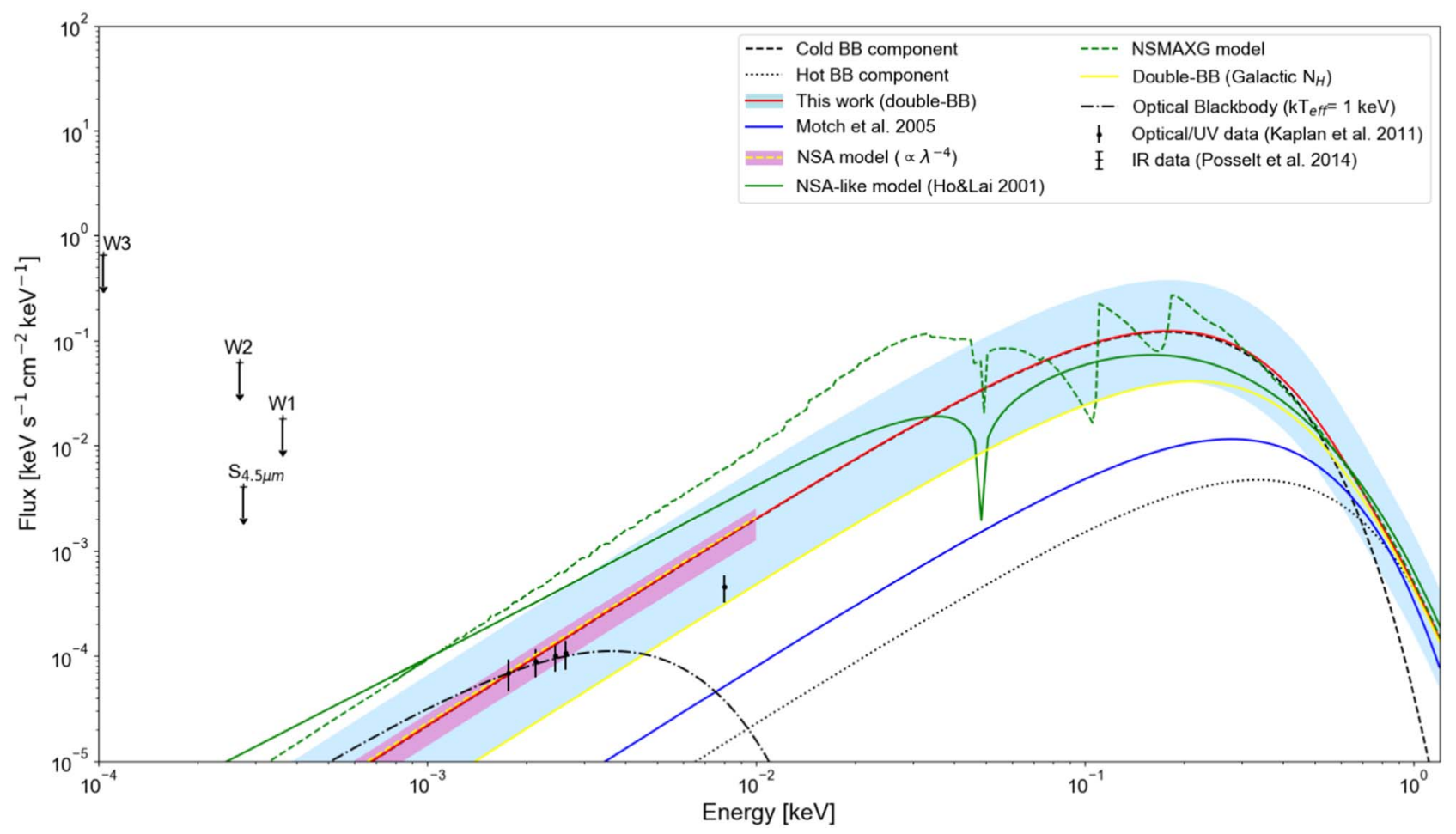

Figure 3. Broadband spectral energy distribution of J1605. The black dotted and dashed lines are the hot and cold (unabsorbed) blackbody components, respectively, obtained from the fit of the X-ray data (see Table 2). The red continuous line represents the sum of the two blackbody components (uncertainty at the $90 \%$ confidence level is shown as the cyan shaded region). The yellow solid line represents the best-fit double-blackbody model obtained with $N_{\mathrm{H}}$ fixed to the Galactic value to the source. For comparison, the best-fit single-blackbody model obtained by Motch et al. (2005) is also shown (continuous blue line). IR upper limits are shown as black arrows. Optical/UV data are shown as black points with error bars, while the black dashed-dotted line represents blackbody emission at $k T_{\text {eff }}=1 \mathrm{keV}$ (see text and Ertan et al. 2017). The best-fit NSA-like model (Ho \& Lai 2001) and NSMAXG model are also reported for comparison (continuous and dashed green lines, respectively). The low-energy tail predicted by the NSA model corrected for a color factor of 2.5 is shown (yellow dashed line), including propagated uncertainty (plum shaded region) in the relevant energy band.

The NSA model provides the effective unredshifted temperature of the NS surface and can be fitted for a few (fixed) values of the magnetic field, while providing the source distance as a free parameter. Our data are well fitted by the NSA model with $B=10^{13} \mathrm{G}$. Similar to the double-blackbody model employed in Section 4.1, the normalization factor $\mathrm{K}_{\mathrm{atmos}}$ of the NSA model is linked to the distance $d$ (in units of parsec) through the relation $K_{\mathrm{NSA}}=1 / d^{2}$, thus resulting in a distance of $92_{-5}^{+5} \mathrm{pc}$, consistent with the results from Section 4.1 and those hinted by Motch et al. (1999, 2005). The surface temperature returned by this model is $\log T_{\text {eff }}=5.737 \pm 0.005 \mathrm{~K}(47.0 \pm 0.5 \mathrm{eV})$.

A constrained configuration of the NSMAXG model has also been found to fit the data. The best-fit NSMAXG model consists of a magnetized $\left(B=10^{13} \mathrm{G}\right) \mathrm{NS}$ at a fixed distance of $100 \mathrm{pc}$ and a normalization value, equal to the ratio of the emitting region compared to the NS radius $\left(R_{\mathrm{em}} / R_{\mathrm{NS}}\right)^{2}$ fixed to unity. This model returns a similar surface temperature as that of the NSA model, $\log T_{\text {eff }}=5.729_{-0.022}^{+0.016} \mathrm{~K}\left(46.2_{-2.3}^{+1.7} \mathrm{eV}\right)$, and a "scaled-up" version of the standard NS, with $M_{\mathrm{NS}}=2.04 M_{\odot}$ and $R_{\mathrm{NS}}=15.6 \mathrm{~km}$ (corresponding to a gravitational redshift of $z_{g}=0.28$ ).

In analogy with Section 4.1, the NSA and NSMAXG models also have been tested with the absorption column density fixed to the Galactic value. However, these models do not fit the data well, leaving enhanced wave-like residuals $\left(\chi_{\text {red }}^{2}=1.4\right)$, especially in the softest $(<0.35 \mathrm{keV})$ part of the spectrum, and will not be further discussed.

Although atmospheric models have been found to satisfactorily fit the X-ray emission of XINS in previous work as well, the temperature values so obtained generally overestimate the observed optical flux by a factor of $\sim 10-100$ (see, e.g., Pavlov et al. 1996; Burwitz et al. 2003; Motch et al. 2003; Pons et al. 2009). For this, we compare the NSA model predictions with the optical/UV data provided in Motch et al. (2005) and Kaplan et al. (2003, 2011), as well as with the doubleblackbody model (see Section 4.1). In fact, at energies softer than the X-ray domain, the blackbody and the NSA models both follow a power law, $F(\lambda) \propto \lambda^{-4}$ (see also Ho et al. 2008). However, the NSA model spectra implemented in XSPEC only extends down to $0.05 \mathrm{keV}$. Furthermore, at $B=10^{13} \mathrm{G}$, the proton cyclotron spectral feature occurs at $0.063 \mathrm{keV}$ and significantly distorts the continuum spectrum near this energy (see Figure 3). This prevents extending the NSA model to optical wavelengths in the form of a power law. Thus, in order to illustrate a fully ionized hydrogen atmosphere spectrum at optical wavelengths, we compute and show an analogous spectrum (i.e., a NSA-like model) using the method described in Ho \& Lai (2001) and values obtained from the NSA best-fit (see Table 2). As shown in Figure 3, the NSA-like model is consistent at optical wavelengths with the double-blackbody model within its relatively large uncertainty. This is still noticeable, as other XINS show high discrepancy between the two models (as reported above). On the other hand, the best-fit NSMAXG model shows a discrepancy from the nominal doubleblackbody at optical wavelengths by a factor of almost 5 . Note that the NSMAXG spectrum shown in Figure 3 takes into account the dense-plasma effect described by Ho et al. (2003), which occurs when photons with a frequency below the local 
plasma frequency have their propagation hindered; this effect causes the spectrum to deviate from a $\lambda^{-4}$, behavior but the precise nature of the deviation is uncertain, therefore the spectrum shown here is not definitive.

Finally, in Figure 3 we also show the low-energy tail predicted by the NSA model $\left(\propto \lambda^{-4}\right)$ approximated as a blackbody spectrum (dashed yellow line) whose temperature is equal to that found by the best-fit model of the NSA component $\left(\log T_{\text {eff }}=5.737 \mathrm{~K}\right.$, see Table 2$)$ and corrected by a color factor of 2.5. ${ }^{21}$ Despite the fact that color factors found by those authors and employed here are computed for nonmagnetic models, it is remarkable how well the resulting spectrum meets the double-blackbody model at optical/UV wavelengths. The NSA model so obtained, similar to the nominal double-blackbody model, is consistent with the optical data but not with the UV data, leading to a possible similar interpretation (see Section 4.1).

\subsection{A pCF Origin for the Absorption Feature}

Spectral absorption features are commonly observed among XINSs. These features are usually attributed to electron or proton cyclotron interactions, and/or to electronic transitions in partially ionized or condensed atmospheres (see Section 4.5). However, absorption features have been also shown to result spuriously as a consequence of fitting, e.g., multi-temperature blackbody emission models (Viganò et al. 2014).

A cyclotron resonant feature is expected in NSs with high $\left(B \geqslant 10^{12} \mathrm{G}\right)$ magnetic fields, where the electron/proton motion perpendicular to the magnetic field lines is quantized in discrete Landau levels, and so are the energies corresponding to those levels, thus resulting in resonant scattering of photons at those energies. The energy of the fundamental line is

$$
E_{\mathrm{CRSF}} \approx \frac{11.6}{\left(1+z_{g}\right)} \frac{m_{e}}{m_{x}} B_{12} \mathrm{keV},
$$

where $B_{12}$ is the magnetic field in units of $10^{12} \mathrm{G}, m_{e}$, and $m_{x}$ are the mass of the electron and that of the particle responsible for the photon scattering, respectively, and $z_{g}$ is the gravitational redshift ( $\sim 0.3$ for standard NS mass and radius).

In our analysis, the absorption feature in the spectrum of $\mathrm{J} 1605$ is found at a nominal energy of $0.432-0.451 \mathrm{keV}$ (depending on the best-fit model, see Table 2). Assuming canonical values for the NS mass and radius $\left(M=1.4 M_{\odot}\right.$ and $R=10 \mathrm{~km})$ and an average value of the absorption line of $0.44 \mathrm{keV}$, the resulting magnetic field is $9.0 \times 10^{13}$ and $4.9 \times 10^{10} \mathrm{G}$ for the proton and electron features, respectively.

Timing studies point out that all other pulsating XINS harbor a magnetic field of the order of $10^{13} \mathrm{G}$, an order of magnitude that is consistent with the results from our fit of the atmospheric model and that inferred by the pCF (see Table 2 and Section 4.3). Moreover, we note that in the case of pCF, the feature is expected to be narrower, with line widths of the order of hundreds of $\mathrm{eV}$ (Nishimura 2003, and references therein), contrary to widths of the order of $\mathrm{keV}$ for eCRSF, although line strength suppression by vacuum polarization in high magnetic fields plays a role in both features (see, e.g., Ho \& Lai 2003). We observe a line width of $\sim 110 \mathrm{eV}$, in agreement with expectations for a $\mathrm{pCF}$ and comparable to the width of

\footnotetext{
${ }^{21}$ This value of the color factor is obtained by Zavlin et al. (1996) for an atmospheric spectrum with the same temperature and composition (pure hydrogen) of the NSA model considered here (see Figure 5 of their work).
}

Gaussian absorption lines in other XINSs whose independent measurements of the magnetic field favor the pCF interpretation (see, e.g., Cropper et al. 2004). Therefore, if the origin of the absorption feature is to be ascribed to cyclotron resonance, our analysis tends to favor the proton rather than the electron as the particle responsible for the scattering/absorption process.

Previous work claimed other absorption features in the spectrum of J1605 that have not been detected in the present work (Haberl 2007; Pires et al. 2014). These features had centroid energies with a 2:3:4 ratio, and were therefore interpreted as the result of harmonic cyclotron features. In Section 3.2 we gave technical reasons that might explain the different results presented in this work. Here, we point out a further physical reason that highlights the difficulty of observing proton harmonic cyclotron resonant features in the spectra of highly magnetic, thermally emitting INSs. In fact, in such physical conditions the strength of each pCF harmonic would scale with the feature centroid energy $E$ as $\sim E / m_{x} c^{2}$ with respect to the fundamental (where $m_{x}$ is the mass of the particle responsible for the scattering). Accordingly, pCFs would result in progressively weaker lines (Schwope et al. 2007; van Kerkwijk \& Kaplan 2007; Potekhin 2010), contrary to the observations. However, we note that quantum effects can make electron cyclotron harmonics stronger than the simple $\sim E / m_{x} c^{2}$ scaling (Suleimanov et al. 2010, 2012), and therefore make them observable. Furthermore, Pires et al. (2019) also found no evidence of the previously reported absorption features.

Finally, as noted by Viganò et al. (2014), surface temperature inhomogeneities can mimic absorption lines, at least in the spectra of some XINSs. Those authors found that a pure blackbody model can result in a spurious absorption feature at $\sim 0.45 \mathrm{keV}$, which instead can be accounted for by a synthetic model composed of several surface temperature distributions. However, the employment of a double-blackbody model should ensure our analysis to be free from spurious detections at $\sim 0.45 \mathrm{keV}$ because this energy value lies close to the Wien peak of one of the blackbody components, thus enhancing the robustness of our results. Nonetheless, a double-blackbody model also can result in spurious absorption features around the energy where the flux from the cold and hot components is comparable. For J1605, our analysis reveals that comparable flux contributions in the double-blackbody model are found between 0.7 and $0.8 \mathrm{keV}$ (see Figure 1), consistent with the centroid energy of the broad absorption line reported in previous works on J1605 (see Section 1). Therefore, we conclude that the claimed broad absorption line at $\sim 0.8 \mathrm{keV}$ in $\mathrm{J} 1605$ is most likely model-dependent.

\subsection{An Atmospheric Origin for the Absorption Feature}

An alternative explanation for the absorption features observed among XINS spectra considers atomic transitions in a hydrogen atmosphere (Lai 2001; Medin \& Lai 2006; van Kerkwijk \& Kaplan 2007; Medin et al. 2008; Potekhin 2014). Absorption features resulting from magnetized atmospheres have been considered in, e.g., Sanwal et al. (2002), van Kerkwijk et al. (2004), and Suleimanov et al. (2012). With a gravitational redshift of $z_{g}=0.3$ (see Section 4.3), and for a mean value of the absorption feature of $0.44 \mathrm{keV}$, the unredshifted energy of the feature goes up to $0.57 \mathrm{keV}$. Considering a partially ionized hydrogen atmosphere (see Section 4.3), such a value does not correspond to expected energies except possibly a transition from ground state to the first excited tightly bound state $(\nu=0$, 
$s=0 \rightarrow 1$, where $\nu, s$ are the principal and magnetic quantum numbers, respectively) and only if $B>10^{13} \mathrm{G}$ (see e.g., Lai 2001; Medin \& Lai 2006; van Kerkwijk \& Kaplan 2007; Ho et al. 2008). Such a high magnetic field is also required for the observed feature to be associated with spectral features due to a condensed surface spectrum (see e.g., Potekhin et al. 2012; Hambaryan et al. 2017). However, for a magnetic field strength as high as that implied by the pCF $\left(9.0 \times 10^{13} \mathrm{G}\right.$, see Section 4.4$)$, absorption features may be washed out due to the vacuum resonance mode conversion (Ho \& Lai 2003; Lai \& Ho 2003; Potekhin et al. 2004; van Adelsberg \& Lai 2006; van Kerkwijk \& Kaplan 2007; Potekhin et al. 2012; Potekhin 2014).

Even though our best-fit atmosphere models are composed of hydrogen, we note that in Medin et al. (2008) there is an expected bound-free transition at $564.9 \mathrm{eV}$ in a helium atmosphere with a magnetic field of $10^{13} \mathrm{G}$. This energy is consistent with the unredshifted feature's nominal energy found by the best-fit double-blackbody model, that is, $0.565 \mathrm{keV}$.

\subsection{Lack of Pulsations}

A periodic X-ray signal associated with the rotation period of J1605 remains undetected. Non-detection of pulsation for this source is also reported by Pires et al. (2019). The upper limit on the pulsed fraction of $\sim 1.3 \%$ we have derived is comparable to the very low modulation fraction of $1.2 \%$ observed for another XINS, RX J1856.5-3754 (Tiengo \& Mereghetti 2007). Such a low level of pulsations indicates that the variations of the apparent thermal flux caused by the changing view of the surface of the NS as it rotates are modest. This could be a consequence of a close alignment between the observer's line of sight and the spin axis of the NS. However, for the case of double-blackbody emission, Pires et al. (2019) argue that the likelihood that we do not see pulsations from the source due to the particularly unfavorable viewing geometry is small, $\sim 2 \%$.

Moreover, or alternatively, the temperature contrast across the stellar surface may be low, or the heat distribution may be approximately symmetric about the rotation axis, which, when combined with relativistic light bending (which acts to suppress the amplitude of thermal pulsations), can result in a low pulsed fraction. At least in the case of the double-blackbody model (see Section 4.1), the temperature contrast between the hot spot and the cooling NS is large, favoring the symmetrical heat distribution interpretation.

On the other hand, the fit of atmospheric models hints to a uniform temperature across the entire surface of the NS (see Section 4.3), which would lead to isotropic emission, and thus the observed lack of pulsation. Although the above represents a tempting scenario, we note that an inhomogeneous temperature distribution is expected across the NS surface due to the presence of strong magnetic fields (see Ho 2007; Page et al. 2007; Pons et al. 2009; Hambaryan et al. 2017, and present work). Nonetheless, other theoretical works (e.g., Potekhin et al. 2003; Kaminker et al. 2006) do not predict strong deviations of the temperature distribution from spherical symmetry, even in the case of strong magnetic fields, e.g., if a "patched" multipole geometry of the magnetic field is present (see Pérez-Azorín et al. 2006).

\section{Summary}

We have performed X-ray spectral and timing analyses of the XINS RX J1605.3+3249 observed with NICER and XMM. Our main results can be summarized as follows:
1. In agreement with Pires et al. (2019), we found no evidence of pulsation for $\mathrm{J} 1605$ with a pulsed fraction greater than $1.3 \%(3 \sigma)$ for periods above $0.15 \mathrm{~s}$. With NICER, we find no pulsations with a pulsed fraction greater than $2.6 \%$ for periods above $2 \mathrm{~ms}$. As such, J1605 remains the only XINS member that exhibits no pulsation. This may be due to either geometrical effects or to isotropic atmospheric emission.

2. The X-ray spectrum of J1605 is equally well fitted by a double-blackbody model and by two magnetic atmospheric models: the NSA/NSA-like model and the NSMAXG model. Double-blackbody and NSA-like models predict consistent flux values at optical wavelengths. The former also fits optical archival data. A color-corrected version of the NSA model also fits the optical data.

3. Those models that fit optical data show a UV excess that, if due to absorption by material surrounding the NS, would require a column density of a few times $10^{21} \mathrm{~cm}^{-2}$. However, IR archival data are difficult to reconcile with this scenario. If the best-fit double-blackbody model with Galactic $N_{\mathrm{H}}$ is considered, UV data are also well fitted, while optical data can be accounted as blackbody emission at $k T_{\text {eff }}=1 \mathrm{keV}$ from a fallback disk.

4. The X-ray spectrum of J1605 shows an absorption feature at $\sim 0.44 \mathrm{keV}$, consistent with prior analyses but without the harmonically related features previously claimed. Our analysis favors the interpretation of this feature as a proton cyclotron resonant feature, implying a magnetic field strength of $9 \times 10^{13} \mathrm{G}$. Contribution to this feature may come from atomic hydrogen transition from the ground state to the first excited tightly bound state.

The lack of a well established value of the distance to J1605 prevents us from inferring additional physical characteristics of this source. It is therefore of key importance to perform further observations in order to measure J1605's distance conclusively.

This work was supported in part by NASA through the NICER mission and the Astrophysics Explorers Program. A portion of the results presented were based on observations obtained with XMMNewton, an ESA science mission with instruments and contributions directly funded by ESA member states and NASA. This research has made use of data and software provided by the High Energy Astrophysics Science Archive Research Center (HEASARC), which is a service of the Astrophysics Science Division at NASA/GSFC and the High Energy Astrophysics Division of the Smithsonian Astrophysical Observatory. We acknowledge extensive use of the NASA Abstract Database Service (ADS) and the ArXiv. C.M. is supported by an appointment to the NASA Postdoctoral Program at the Marshall Space Flight Center, administered by Universities Space Research Association under contract with NASA. W.C.G.H. appreciates use of computer facilities at KIPAC.

Facilities: NICER, XMM-Newton.

\section{ORCID iDs}

Christian Malacaria (ib https://orcid.org/0000-0002-0380-0041 Slavko Bogdanov (iD https://orcid.org/0000-0002-9870-2742 Wynn C. G. Ho (iD https://orcid.org/0000-0002-6089-6836 Teruaki Enoto (i) https://orcid.org/0000-0003-1244-3100 Paul S. Ray (iD https://orcid.org/0000-0002-5297-5278 Sebastien Guillot (i) https://orcid.org/0000-0002-6449-106X 
Tolga Güver (1) https://orcid.org/0000-0002-3531-9842 Gaurava K. Jaisawal (i) https://orcid.org/0000-00026789-2723

Michael T. Wolff (10 https://orcid.org/0000-0002-4013-5650

\section{References}

Anders, E., \& Grevesse, N. 1989, GeCoA, 53, 197

Arnaud, K. A. 1996, in ASP Conf. Ser. 101, Astronomical Data Analysis Software and Systems V, ed. G. H. Jacoby \& J. Barnes (San Francisco, CA: ASP), 17

Borghese, A., Rea, N., Coti Zelati, F., et al. 2017, MNRAS, 468, 2975

Borghese, A., Rea, N., Coti Zelati, F., Tiengo, A., \& Turolla, R. 2015, ApJL, 807, L20

Buccheri, R., Bennett, K., Bignami, G. F., et al. 1983, A\&A, 128, 245

Burwitz, V., Haberl, F., Neuhäuser, R., et al. 2003, A\&A, 399, 1109

Cropper, M., Haberl, F., Zane, S., \& Zavlin, V. E. 2004, MNRAS, 351, 1099

De Luca, A., Caraveo, P. A., Mereghetti, S., Negroni, M., \& Bignami, G. F. 2005, ApJ, 623, 1051

Ertan, Ü., Calışkan, S., \& Alpar, M. A. 2017, MNRAS, 470, 1253

Foight, D. R., Güver, T., Özel, F., \& Slane, P. O. 2016, ApJ, 826, 66

Gänsicke, B. T., Braje, T. M., \& Romani, R. W. 2002, A\&A, 386, 1001

Gendreau, K. C., Arzoumanian, Z., Adkins, P. W., et al. 2016, Proc. SPIE, 9905, 99051H

Gotthelf, E. V., \& Halpern, J. P. 2009, ApJL, 695, L35

Haberl, F. 2007, Ap\&SS, 308, 181

Haberl, F., Schwope, A. D., Hambaryan, V., Hasinger, G., \& Motch, C. 2003, A\&A, 403, L19

Halpern, J. P., \& Wang, F. Y.-H. 1997, ApJ, 477, 905

Hambaryan, V., Suleimanov, V., Haberl, F., et al. 2017, A\&A, 601, A108

Hambaryan, V., Suleimanov, V., Schwope, A. D., et al. 2011, A\&A, 534, A74

Ho, W. C. G. 2007, MNRAS, 380, 71

Ho, W. C. G., \& Heinke, C. O. 2009, Natur, 462, 71

Ho, W. C. G., Kaplan, D. L., Chang, P., Van Adelsberg, M., \& Potekhin, A. Y. 2007, MNRAS, 375, 821

Ho, W. C. G., \& Lai, D. 2001, MNRAS, 327, 1081

Ho, W. C. G., \& Lai, D. 2003, MNRAS, 338, 233

Ho, W. C. G., Lai, D., Potekhin, A. Y., \& Chabrier, G. 2003, ApJ, 599, 1293

Ho, W. C. G., Potekhin, A. Y., \& Chabrier, G. 2008, ApJS, 178, 102

Ho, W. C. G. 2014, in IAU Symp. 302, Magnetic Fields throughout Stellar Evolution, ed. P. Petit, M. Jardine, \& H. C. Spruit (Cambridge: Cambridge Univ. Press), 435

Hohle, M. M., Haberl, F., Vink, J., de Vries, C. P., \& Neuhäuser, R. 2012, MNRAS, 419, 1525

Jahoda, K., Markwardt, C. B., Radeva, Y., et al. 2006, ApJS, 163, 401

Jethwa, P., Saxton, R., Guainazzi, M., Rodriguez-Pascual, P., \& Stuhlinger, M. 2015, A\&A, 581, A104

Kaminker, A. D., Yakovlev, D. G., Potekhin, A. Y., et al. 2006, MNRAS, 371, 477

Kaplan, D. L. 2008, in AIP Conf. Ser. 968, Astrophysics of Compact Objects, ed. Y.-F. Yuan, X.-D. Li, \& D. Lai (Melville, NY: AIP), 129

Kaplan, D. L., Kamble, A., van Kerkwijk, M. H., \& Ho, W. C. G. 2011, ApJ, 736, 117

Kaplan, D. L., Kulkarni, S. R., \& van Kerkwijk, M. H. 2003, ApJL, 588, L33

Kaspi, V. M., Roberts, M. S. E., \& Harding, A. K. 2006, in Isolated Neutron Stars, ed. W. H. G. Lewin \& M. van der Klis (Cambridge: Cambridge Univ. Press), 279

Lai, D. 2001, RvMP, 73, 629

Lai, D., \& Ho, W. C. G. 2003, ApJ, 588, 962

Medin, Z., \& Lai, D. 2006, PhRvA, 74, 062507

Medin, Z., Lai, D., \& Potekhin, A. Y. 2008, MNRAS, 383, 161

Morrison, R., \& McCammon, D. 1983, ApJ, 270, 119

Motch, C., Haberl, F., Zickgraf, F.-J., Hasinger, G., \& Schwope, A. D. 1999, A\&A, 351, 177
Motch, C., Pires, A. M., Haberl, F., Schwope, A., \& Zavlin, V. E. 2009, A\&A, 497, 423

Motch, C., Sekiguchi, K., Haberl, F., et al. 2005, A\&A, 429, 257

Motch, C., Zavlin, V. E., \& Haberl, F. 2003, A\&A, 408, 323

Nishimura, O. 2003, PASJ, 55, 849

Page, D., Geppert, U., \& Küker, M. 2007, Ap\&SS, 308, 403

Page, D., Geppert, U., \& Weber, F. 2006, NuPhA, 777, 497

Page, D., Lattimer, J. M., Prakash, M., \& Steiner, A. W. 2004, ApJS, 155, 623

Pavlov, G. G., Shibanov, Y. A., Zavlin, V. E., \& Meyer, R. D. 1995, in NATO Advanced Science Institutes (ASI) Series C, Vol. 450, ed. M. A. Alpar, U. Kiziloglu, \& J. van Paradijs (Dordrecht: Kluwer), 71

Pavlov, G. G., Zavlin, V. E., Truemper, J., \& Neuhaeuser, R. 1996, ApJL, 472, L33

Pérez-Azorín, J. F., Pons, J. A., Miralles, J. A., \& Miniutti, G. 2006, A\&A, 459, 175

Perna, R., Hernquist, L., \& Narayan, R. 2000, ApJ, 541, 344

Pires, A. M., Haberl, F., Zavlin, V. E., et al. 2014, A\&A, 563, A50

Pires, A. M., Schwope, A. D., Haberl, F., et al. 2019, A\&A, 623, A73

Pons, J. A., Miralles, J. A., \& Geppert, U. 2009, A\&A, 496, 207

Popov, S. B., Colpi, M., Prokhorov, M. E., Treves, A., \& Turolla, R. 2003, A\&A, 406, 111

Posselt, B., Pavlov, G. G., Ertan, Ü., et al. 2018, ApJ, 865, 1

Posselt, B., Pavlov, G. G., Popov, S., \& Wachter, S. 2014, ApJS, 215, 3

Posselt, B., Popov, S. B., Haberl, F., et al. 2007, Ap\&SS, 308, 171

Potekhin, A. Y. 2010, A\&A, 518, A24

Potekhin, A. Y. 2014, PhyU, 57, 735

Potekhin, A. Y., De Luca, A., \& Pons, J. A. 2015, SSRv, 191, 171

Potekhin, A. Y., Ho, W. C. G., \& Chabrier, G. 2016, arXiv:1605.01281

Potekhin, A. Y., Lai, D., Chabrier, G., \& Ho, W. C. G. 2004, ApJ, 612, 1034

Potekhin, A. Y., Suleimanov, V. F., van Adelsberg, M., \& Werner, K. 2012, A\&A, 546, A121

Potekhin, A. Y., Yakovlev, D. G., Chabrier, G., \& Gnedin, O. Y. 2003, ApJ, 594, 404

Prigozhin, G., Gendreau, K., Foster, R., et al. 2012, Proc. SPIE, 8453, 845318

Ransom, S. M., Gaensler, B. M., \& Slane, P. O. 2002, ApJL, 570, L75

Sanwal, D., Pavlov, G. G., Zavlin, V. E., \& Teter, M. A. 2002, ApJL, 574, L61

Schwope, A. D., Hambaryan, V., Haberl, F., \& Motch, C. 2007, Ap\&SS, 308,619

Schwope, A. D., Hasinger, G., Schwarz, R., Haberl, F., \& Schmidt, M. 1999, A\&A, 341, L51

Staubert, R., Klochkov, D., Vybornov, V., Wilms, J., \& Harrison, F. A. 2016 A\&A, 590, A91

Strüder, L., Briel, U., Dennerl, K., et al. 2001, A\&A, 365, L18

Suleimanov, V. F., Pavlov, G. G., \& Werner, K. 2010, ApJ, 714, 630

Suleimanov, V. F., Pavlov, G. G., \& Werner, K. 2012, ApJ, 751, 15

Tetzlaff, N., Schmidt, J. G., Hohle, M. M., \& Neuhäuser, R. 2012, PASA, 29,98

Tiengo, A., \& Mereghetti, S. 2007, ApJL, 657, L101

Turner, M. J. L., Abbey, A., Arnaud, M., et al. 2001, A\&A, 365, L27

Turolla, R. 2009, in Astrophysics and Space Science Library, Vol. 357, ed W. Becker (Berlin: Springer), 141

van Adelsberg, M., \& Lai, D. 2006, MNRAS, 373, 1495

van Kerkwijk, M. H., \& Kaplan, D. L. 2007, Ap\&SS, 308, 191

van Kerkwijk, M. H., Kaplan, D. L., Durant, M., Kulkarni, S. R., \& Paerels, F. 2004, ApJ, 608, 432

Viganò, D., Perna, R., Rea, N., \& Pons, J. A. 2014, MNRAS, 443, 31

Walter, F. M. 2001, ApJ, 549, 433

Wilms, J., Allen, A., \& McCray, R. 2000, ApJ, 542, 914

Zane, S., Cropper, M., Turolla, R., et al. 2005, ApJ, 627, 397

Zane, S., Haberl, F., Israel, G. L., et al. 2011, MNRAS, 410, 2428

Zavlin, V. E., \& Pavlov, G. G. 2002, in Proc. of the 270 WE-Heraeus Seminar on Neutron Stars, Pulsars, and Supernova Remnants, MPE Report 278, ed.

W. Becker, H. Lesch, \& J. Trümper (Garching: Max-Plank-Institut für Extraterrestrische Physik), 263

Zavlin, V. E., Pavlov, G. G., \& Shibanov, Y. A. 1996, A\&A, 315, 141 